\title{
Parameter Discussion of Bearing Capacity Analysis of Highway Bridges in Heavy-cargo Transportation
}

\author{
Ping $\mathrm{Lu}^{1, \mathrm{a}}$, Haiyang $\mathrm{Yu}^{2, \mathrm{~b}}$, Li He ${ }^{1, \mathrm{c}}$, Haijun $\mathrm{Wu}^{1, \mathrm{~d}}$ \\ ${ }^{1}$ School of Civil Engineering and Architecture, Chongqing Jiaotong University, Chongqing, China \\ ${ }^{2}$ Construction Engineering Quality Supervision Station of Jiangjin District of Chongqing, China \\ a58392137@qq.com, b352276628@qq.com, c1535051259@qq.com, d583921237@qq.com
}

Keywords: Bridge engineering; Heavy-cargo transportation; Transverse distribution; Impact coefficient; Temperature

Abstract: To analyze and discuss about selection of parameters of bearing capacity calculation when heavy-cargo transportation pass through bridges, characteristics of heavy-cargo transportation significantly different from general vehicle design load, is analyzed. According to the characteristics of the operation and management of heavy-cargo transportation, the most unfavorable condition of heavy-cargo transportation on bridge deck is analyzed by using dynamic programming principle, and value-taking method of transverse distribution coefficient of heavycargo transportation is proposed. Finally, the rationality of this method is verified by example calculation.

\section{Introduction}

The heavy-cargo transportation load has many characteristics such as the load level, load distribution, the frequency of oversize transportation, traffic conditions, which obviously are different from general automobile load. So, the researching relevant parameters of the bridge bearing capacity calculation under the effect of heavy-cargo transportation have crucial practical significance ${ }^{[1]}$.

\section{The basic characteristics of heavy-cargo transportation}

Heavy-cargo transportation load, with low emerging frequency and short duration, is temporary and occasional for load standard of highway bridge design. Heavy-cargo transportation is different from general vehicle transportation with the following salient features ${ }^{[2,3,4,5]}$ :

(1) Coercive characteristic of heavy-cargo transportation.

(2) Low emerging frequency of heavy-cargo transportation load.

(3) Heavy-cargo transportation could pass through the bridge before declaration and approval of competent authority.

(4) Heavy-cargo transportation must run according to the time and the route of approval agency appointing.

(5) Speed of heavy-cargo transportation is strictly limited when running.

(6) Heavy-cargo transportation often acts on the bridge without other vehicles together.

\section{Transverse distribution coefficient calculation of load in heavy-cargo transportation}

Analysis of the most unfavorable loading position of load in transverse. In the same bridge, transverse distribution coefficient, called $m^{[6]}$, of each piece of main beams is different, and different type of load is so. Because of structural particularity of oversize vehicles, its parameters, number of wheels and wheel track, determine transverse arrangement on bridge deck and effect of load on highway bridges. Moreover, it is difficult that oversize vehicles run along the centerline of the bridge accurately. So, it is unreasonable that control standard of load is determined directly by the arrangement — oversize vehicles in the middle of bridge deck. When calculating control standard of load of heavy-cargo transportation, we should, firstly, determine the most unfavorable condition, and we can get reasonable control standard value of load by calculating the transverse distribution 
coefficient.

Principle of the most unfavorable loading position in transverse. We can get the extreme value of influence quantity and position of the extreme value quickly by dynamically loading influence line when loading in transverse. It's method as follows:

As shown in Figure 1, $f(x)$ is influence line of intervals, $[B, E]$, of cross section. We define $U(x)$ as the maximum, called extremal function, when loading in the intervals $[E, x]$. It is the maximum possible loading in the intervals $[E, x]$. Because $U(x)$ is monotone non-decreasing function,' we cannot get the maximum loading in horizontal section of the curve, and $U(E)$ which is in the far right represents the maximum of internal force after loading in intervals of influence line $e^{[7]}$
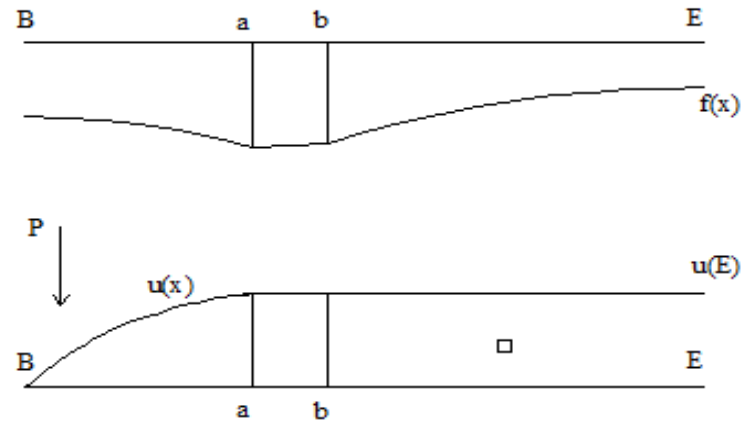

Figure 1. To calculate the maximum of internal force by using dynamic programming

According to computing for bridge structures, we can write down the recurrence formula about $U_{i}(x)-i$ represents numbers of car in transverse. We put the influence line of interval into a plurality of equally spaced points; moreover, the value of $\Delta x$ must be an integer multiple of the wheel track.

Firstly, we write down the recurrence formula of $U_{1}$ :

In formula, number of point represent length; point $B$ is 1 and point $E$ is $n$. When $x$ at point $B$, the value of $U_{1}(x)$ as follows:

$$
U_{1}(B)= \begin{cases}g(B) & g(B)>0 \\ 0 & g(B) \leq 0\end{cases}
$$

When $B+1 \leq x \leq B+e_{1}-1$, recurrence formula as follows

$$
U_{1}(x)= \begin{cases}g(x) & g(x)>U_{1}(x-1) \\ U_{1}(x-1) & g(x) \leq U_{1}(x-1)\end{cases}
$$

In the above formula, $e_{1}$ is distance that wheels move on the transverse influence line.

If there are multiple vehicles in transverse, $U_{n}(x)$, the extreme value curve of $\mathrm{n}$ cars, is derived by the formula as follows:

When $B \leq x \leq(n-1) * a_{1}-1\left(a_{1}\right.$ is transverse distance of wheel)

$$
U_{n}(x)=U_{n-1}(x)
$$

When $B+(n-1) * a_{1} \leq x \leq B+e_{1}-1$ 


$$
U_{n}(x)= \begin{cases}g(x)+U_{n-1}\left(x-a_{1}\right) & g(x)+U_{n-1}\left(x-a_{1}\right)>U_{n}(x-1) \\ U_{n}(x-1) & g(x)+U_{n-1}\left(x-a_{1}\right) \leq U_{n}(x-1)\end{cases}
$$

According to the above formulas, we can obtain the curve about extreme value of loads of oversize vehicle. Thus we can calculate the transverse distribution coefficient of load of oversize vehicles.

\section{An example of calculation}

Taking a bridge, span is $20 \mathrm{~m}$, which is made of 10 hinged prestressed concrete hollow slabs, as an example, we obtain the most unfavorable position of oversize vehicles by dynamic programming method. Because the influence line of hinged slab bridge is symmetric, this paper only analyzes No. 1 5 main beams.

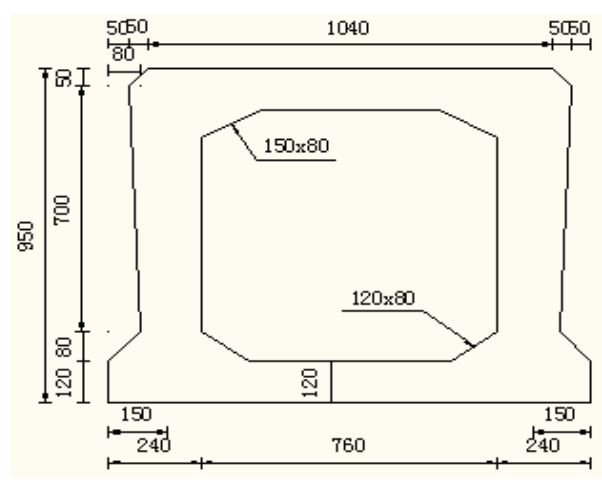

Figure 2. Cross section of $20 \mathrm{~m}$ hollow slabs bridge [Unit: $\mathrm{mm}$ ]

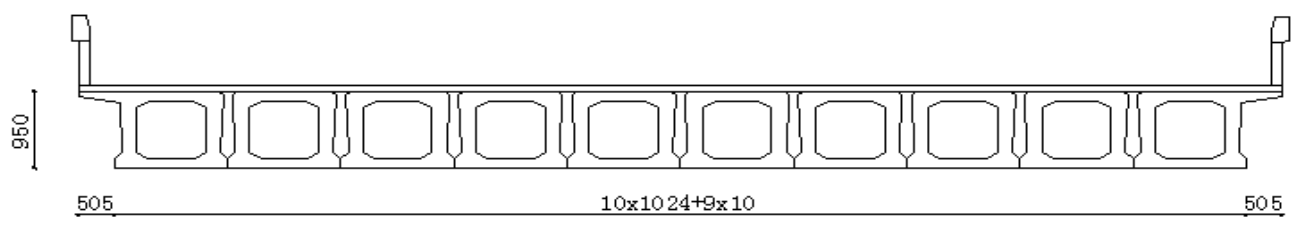

Figure 3. Superstructure of $20 \mathrm{~m}$ hollow slabs bridge

Figure 2 and Figure 3 represent cross section of hollow slab. Using Midas/civil sectional characteristics calculator, this paper got the answer: bending moment of inertia $I=0.0638 \mathrm{~m}^{4}$, torsion moment of inertia $I_{T}=0.1153 \mathrm{~m}^{4}$. The transverse distance of wheels of identified two column of oversize vehicles is $0.9 \mathrm{~m}+1.2 \mathrm{~m}+0.9 \mathrm{~m}$. According to dynamic programming principle, taking value $\Delta x=0.1 m$, when left offset distance that wheels are on the deck is $e_{1}=1$, transverse distribution coefficient of each piece of main beams is shown in Table 1 and Table 2.

Table 1. Transverse distribution coefficient of the middle beam when position which oversize vehicles load is different

\begin{tabular}{|c|c|c|c|c|c|}
\hline Beam number & NO.1 & NO.2 & NO.3 & NO.4 & NO.5 \\
\hline Loading position & 0.0849 & 0.0891 & 0.0977 & 0.1097 & 0.1181 \\
\hline Lenterline of the deck & 0.0862 & 0.0904 & 0.0991 & 0.1108 & 0.1185 \\
\hline Left eccentricity 0.1m & 0.0875 & 0.0917 & 0.1005 & 0.112 & 0.1189 \\
\hline Left eccentricity 0.3m & 0.0888 & 0.093 & 0.1019 & 0.1131 & 0.1193 \\
\hline Left eccentricity 0.4m & 0.0901 & 0.0944 & 0.1034 & 0.1143 & 0.1198 \\
\hline Left eccentricity 0.5m & 0.0914 & 0.0958 & 0.1049 & 0.1155 & 0.1203 \\
\hline Left eccentricity $0.6 \mathrm{~m}$ & 0.0928 & 0.0972 & 0.1065 & 0.1166 & 0.1208 \\
\hline
\end{tabular}


Table 2. Transverse distribution coefficient of the middle beam when position which oversize vehicles load is different(continued)

\begin{tabular}{|c|c|c|c|c|c|}
\hline Beam number & NO.1 & NO.2 & NO.3 & NO.4 & NO.5 \\
\hline Left eccentricity $0.7 \mathrm{~m}$ & 0.0942 & 0.0988 & 0.1082 & 0.1177 & 0.1208 \\
\hline Left eccentricity $0.8 \mathrm{~m}$ & 0.0956 & 0.1003 & 0.1099 & 0.1186 & 0.1204 \\
\hline Left eccentricity 0.9m & 0.0971 & 0.1019 & 0.1116 & 0.1195 & 0.1201 \\
\hline Left eccentricity 1.0m & 0.0986 & 0.1035 & 0.1133 & 0.1204 & 0.1199 \\
\hline
\end{tabular}

when left offset distance that wheels are on the deck is $e_{1}=1$, transverse distribution coefficient of each piece of main beams as the figure 4 shows. The $m$ of main beams, excepted NO.5 beam, as increasing distance that vehicles deviate from the deck centerline increase monotonically. Transverse distribution coefficient of NO.5 beam increases firstly and decreases later; variation of it as the figure 4 shows.

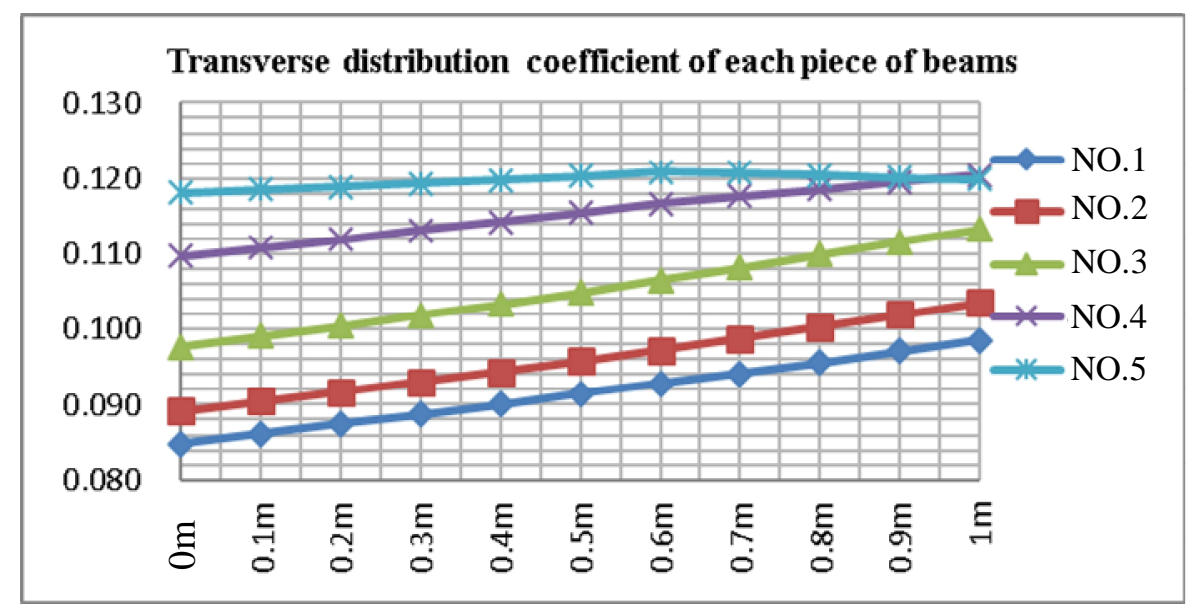

Figure 4. Transverse distribution coefficient of each main beam varies with the position of oversize vehicles

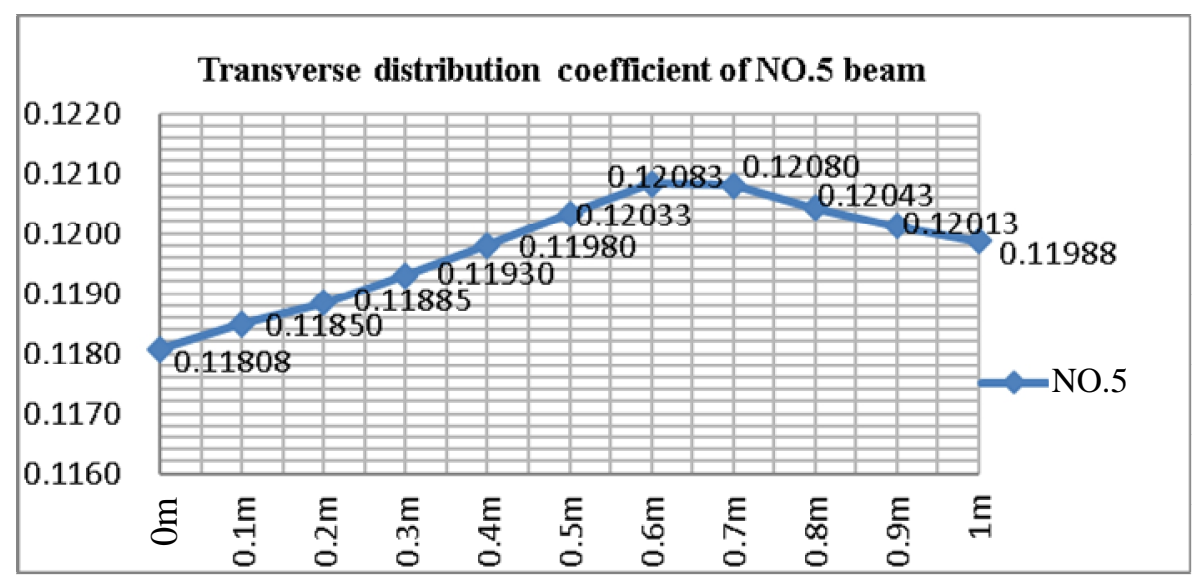

Figure 5. Transverse distribution coefficient of No.5 beam varies with the position of oversize vehicles

Above Figure 5, The $m$ of No.5 beam decreases after an initial increase; the value of $m$ decreases gradually after it is the maximum when the offset distance is 0.6 meters. The gap of transverse distribution coefficient is $2.24 \%$ between vehicles in middle and eccentricity equal 0.6 meters; it is small. As the continued increase of, load will be gradually transferred to the No.4 beam and replace the No.5 beam. Therefore, considering number of the oversize vehicle's wheels in transverse is a few more, we can approximately take the larger one between traveling in middle and in eccentric equal 0.5 meters about transverse distribution coefficient. The vehicles that is allowed to traffic 
according control standard of load should travel in middle when passing bridge, transverse deviation of the vehicle on the deck should be controlled within a range of \pm 0.5 meters.

\section{Conclusion}

This study mainly discussed that calculation parameters are selected during evaluating bearing capacity when oversize vehicles pass existing bridge; conclusion as follows:

(1) Heavy-cargo transportation load, with low emerging frequency and short duration, is temporary and occasional for load standard of highway bridge designing. Heavy-cargo transportation is usually under the close supervision, and the timing of its passage and the arrangement of the load can be accommodated and controlled; because of that, the selection of parameters is different from general when calculating.

(2) Oversize vehicle structure and parameters, number of wheels and wheel track, determine transverse arrangement on bridge deck and effect of load on highway bridges. Moreover, it is difficult that oversize vehicles run along the centerline of the bridge accurately. So, it is unreasonable that control standard of load is determined directly by the arrangement-oversize vehicles in the middle of bridge deck. According to using dynamic programming principle, the ideal and method, solving the most unfavorable loading position in transverse, is suggested. And by practical examples, this paper reasonably solute the problem about calculating transverse distribution of oversize vehicles on the deck.

\section{Acknowledgements}

This paper is supported by Traffic science and technology project of Chongqing City (No. 2012(4)), Scientific Projects of State Key Laboratory Breeding Base of Mountain Bridge and Tunnel Engineering (CQSLBF-Y14-12; CQSLBF-Y15-7), the Fundamental Research Funds for the Central Universities (No.310821161120) and Scientific Projects of the engineering research center of bridge structure and material in the mountain area, Ministry of Education, Chongqing Jiaotong University.

\section{References}

[1] Study on the Large-cargo Transportation Load Control Standard and Countermeasures of Chongqing Highway[R]. Chongqing: Chongqing Communications Planning Survey \& Design Institute, 2014.

[2] Yu Haiyang. Study on the Large-cargo Transportation Analysis Methods and Control Standard of Conventional Bridge on Chongqing Highway[D]. Chongqing: Chongqing Jiaotong University, 2014.

[3] General Administration of Quality Supervision, Inspection and Quarantine of the People's Republic of China, Standardization Administration of the People's Republic of China. GB15892004. Limits of Dimensions, Axle Load and Masses for Road Vehicles[S]. Beijing: China Communication Press, 2004.

[4] Ministry of Transport of the People's Republic of China. JTG H11-2004. Code for Maintenance of Highway Bridges and Culvers[S]. Beijing: China Communication Press, 2004.

[5] Ministry of Transport of the People's Republic of China. JTG D60-2004. General Specifications for Design of Highway Bridge and Culverts[S]. Beijing: China Communication Press, 2004.

[6] Fan Lichu. Bridge Engineering[M]. Beijing: China Communication Press, 2012.

[7] Shi Dong, Shi Zhiyuan, Huang Dongzhou. Computing for Bridge Structures[M]: Shanghai: Tongji University Press, 1987.84-103. 